\title{
Benthaminin 3, a Novel Antibacterial Cassane-Type Furanoditerpenoid from Caesalpinia benthamiana
}

\author{
Rita A. Dickson ${ }^{*, 1}$, Kofi Annan ${ }^{1}$, Theophilus C. Fleischer ${ }^{1}$ and Peter J. Houghton ${ }^{2}$ \\ ${ }^{1}$ Pharmacognosy Department, Faculty of Pharmacy and Pharmaceutical Sciences, Kwame Nkrumah \\ University of Science and Technology, Ghana \\ ${ }^{2}$ Pharmacognosy Research Laboratories, Pharmaceutical Sciences Research Division, King's College \\ London, Franklin-Wilkins Building, 150 Stamford Street, London SE1 9NH, UK
}

\begin{abstract}
The roots of Caesalpinia benthamiana (synonym. Mezoneuron benthamianum) are considered to be an effective remedy in Ghana for the management of skin diseases and wounds. Bioactivity-guided fractionation of the chloroform extract of the root bark of the plant has resulted in the isolation of a novel cassane-type furanoditerpenoid, designated as benthaminin 3. The structure of the compound was elucidated by the use of spectroscopic techniques. The antibacterial activity of the compound has been assessed using the microdilution assay method. The lowest MIC $(63 \mu \mathrm{g} / \mathrm{mL})$ was recorded against Staphylococcus aureus and Bacillus subtilis. The compound was also observed to have a mild inhibitory effect against resistant strains of bacteria including methicillin-resistant $S$. aureus, tetracyline-resistant $S$. aureus and erythromycin-resistant $S$. aureus with MIC values greater than $1000 \mu \mathrm{g} / \mathrm{mL}$.
\end{abstract}

Keywords: Caesalpinia benthamiana (Baill.) Herend. and Zarucchi, Mezoneuron benthamianum Baill, Caesalpinaceae, Cassane-type furanoditerpenoids, Antibacterial activities.

\section{INTRODUCTION}

Caesalpinia benthamiana (Baill.) Herend. and Zarucchi (syn. Mezoneuron benthamianum Baill.) (Caesalpinaceae) [1] is a shrub found mostly in secondary forest in Ghana and finds use in the treatment of topical infections and wounds $[2,3]$. Traditionally, a paste made from powdered root bark mixed with shea butter, palm oil or palm kennel oil may be used topically [4]. Plants belonging to the genus Caesalpinia are a rich source of cassane-type furanoditerpenoids which are reported to possess a number of biological activities e.g. antiplasmodial [5], antiviral [6] and anti-diarrhoeal effects [7].

In a previous study of this species we reported the isolation and characterization of two new cassane diterpenoids, benthaminins 1 and 2, from the active fractions of the petroleum spirit extract possessing antibacterial and antioxidant activities [8]. A third compound was also isolated which was a deoxy form of caesaldekarin C (also referred to as methyl vouacapenate) which had previously been isolated from Caesalpinia major, C. bonducella, Vouacapoua americana and $V$. macropetala $[9,10]$.

In a continuation of this research the antibacterial properties of the chloroform extract of the root bark of $C$. benthamianum were investigated using antimicrobial assay procedures.

*Address corresponding to this author at the Pharmacognosy Department, Faculty of Pharmacy and Pharmaceutical Sciences, Kwame Nkrumah University of Science and Technology, Ghana; Tel: +233204620000;

Fax: +233(0)63919; E-mail: radickson.pharm@knust.edu.gh

\section{RESULTS AND DISCUSSION}

\subsection{Isolation and Purification of Compound RC4 (Designated Benthaminin 3)}

Bioactivity-guided analysis was carried out using agar overlay bioautography on six fractions (MCF1MCF6) obtained from silica gel CC of the chloroform extract of $C$. benthamiana. MCF2 and MCF6 were found to be active against $B$. subtilis.

Further fractionation of MCF6 on column chromatography (SG/DCM:MeOH) yielded fractions MCF21-MCF25.

MCF25 was subjected to further repeated column chromatography on silica to afford a pure compound $\mathrm{RC} 4$, eventually designated benthaminin $3(33 \mathrm{mg})$. The structure of the compound was elucidated using a combination of $1 \mathrm{D}$ and 2D NMR spectroscopy, mass spectrometry (LREIMS, HREIMS and ESI) and reference to the literature $[8,9,11]$.

\subsubsection{Compound RC4}

Benthaminin 3 (RC4) was obtained as white amorphous compound; LREIMS $\mathrm{m} / \mathrm{z}$ 384. See Table 1 for ${ }^{1} \mathrm{H}$ and ${ }^{13} \mathrm{C}$ NMR data. It had molecular formula $\mathrm{C}_{23}$ $\mathrm{H}_{28} \mathrm{O}_{5}$ based on HREIMS spectral analysis of $\mathrm{m} / \mathrm{z} 384$. The ${ }^{1} \mathrm{H}$ NMR spectrum (Table 1 ) is similar to that of deoxycaesaldekarin $C$ previously isolated from the petroleum spirit extract of the same plant $[8,9,11]$ apart from the presence of an additional acetyl group shown by the methyl signal at $\delta 2.33$ and the fact that 
Table 1: ${ }^{1} \mathrm{H}$ NMR and ${ }^{13} \mathrm{C}$ NMR Spectroscopic Data $(\delta, \mathrm{Hz})$ of Benthaminin 3 in $\mathrm{CDCl}_{3}$

\begin{tabular}{|c|c|c|}
\hline Position & ${ }^{1}$ H Chemical shifts (ppm) & ${ }^{13} \mathrm{C}$ Chemical shifts (ppm) \\
\hline $1 \alpha$ & $1.56(\mathrm{~m})$ & 36.5 \\
\hline $1 \beta$ & $1.77(m)$ & \\
\hline $2 \alpha$ & $1.57(\mathrm{~m})$ & 18.1 \\
\hline $2 \beta$ & $1.65(m)$ & \\
\hline $3 \alpha$ & $1.29(m)$ & 38.4 \\
\hline $3 \beta$ & $1.74(m)$ & \\
\hline 4 & & 48.0 \\
\hline 5 & $1.90(\mathrm{~m})$ & 42.1 \\
\hline 6 & $5.30(s)$ & 70.7 \\
\hline $7 \alpha$ & $2.95(m)$ & 21.7 \\
\hline $7 \beta$ & $2.99(m)$ & \\
\hline 8 & & 128.7 \\
\hline 9 & & 145.5 \\
\hline 10 & & 38.0 \\
\hline 11 & $6.72(s)$ & 105.0 \\
\hline 12 & & 153.8 \\
\hline 13 & & 123.8 \\
\hline 14 & & 125.8 \\
\hline 15 & $6.73(s)$ & 106.6 \\
\hline 16 & $7.26(s)$ & 144.5 \\
\hline 17 & & 24.3 \\
\hline 18 & $1.45(m)$ & 16.5 \\
\hline 19 & & 178.6 \\
\hline 20 & $1.25(m)$ & 16.0 \\
\hline 21 & $3.72(s)$ & 52.3 \\
\hline 22 & & 170.8 \\
\hline 23 & $2.33(s)$ & 18.9 \\
\hline
\end{tabular}

ring $\mathrm{C}$ was aromatic as shown by carbon signals at $\delta \mathrm{c}$ 128.7(C-8), 145.5 (C-9), 105.0 (C-11), 153.8 (C-12), 123.8 (C-13), 125.8 (C-14), 106.6 (C-15) and 144.5 (C16). RC4 also showed resonances for the 2,3disubstituted furan at $\delta 7.26(s)$ and $\delta 6.73(s)$, downfield from the corresponding positions in the spectrum of deoxycaesaldekarin $\mathrm{C}$, indicating a more electronegative environment. This was explained by the fact that the adjacent ring was aromatic in nature, as shown by the shifts of the relevant $\mathrm{C}$ atoms

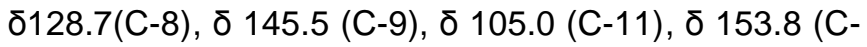
12), $\delta 123.8$ (C-13) and $\delta 125.8$ (C-14), similar to those shown by benthaminin 1 isolated previously [8]. This was confirmed by HMBC studies which showed the aromatic proton at $\delta 6.73$ was directly attached to a carbon resonating at $\delta 105.0$ (assigned to C-11). This proton also showed HMBC correlations to aromatic carbons at $\delta 123.8 \quad(\mathrm{C}-13), \quad 8128.7 \quad(\mathrm{C}-8)$ and a quaternary carbon at $\delta 38.0(\mathrm{C}-10)$. The presence of an additional aromatic singlet at $\delta 6.73(\mathrm{H}-15)$ and a methyl attached to an aromatic ring ( $\delta 1.45)$ confirmed the presence of an aromatic ring of a caesaldekarin type. Two $3 \mathrm{H}$ singlets appeared at $\delta 3.72$ and $\delta 2.33$ indicated the presence of a methoxy ester and acetyl groups. These data are broadly consistent with the structure being an aromatized form of deoxycaesaldekarin C, analogous to benthaminin 1 but with an additional acetyl group. The additional acetyl group is considered to be attached at C-6 ( $\delta \mathrm{c} 70.8)$, with the $6-\mathrm{H}$ resonating at $\delta 5.3$, which is similar to the signal observed in caesalmin C [6]. The methyl group signal at $\delta 1.25$ had HMBC correlations to the ester carbonyl signal at $\delta 178.6$ (C-19), the quaternary carbon at $\delta 48.0$ (C-4) and a methylene carbon at $\delta 38.4$ (assigned to C-3). 
NOESY correlations are as in caesalmin $C$ and deoxycaesaldekarin $C[6,8]$.

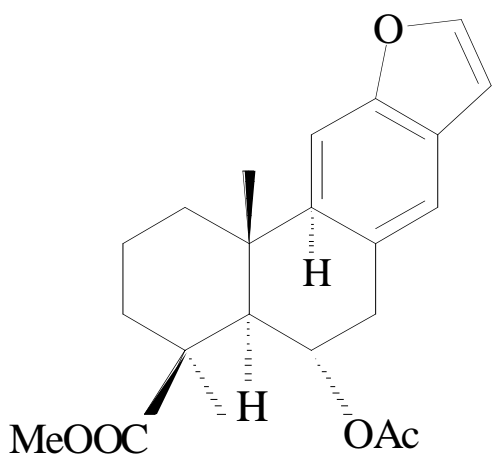

Figure 1: Benthaminin 3.

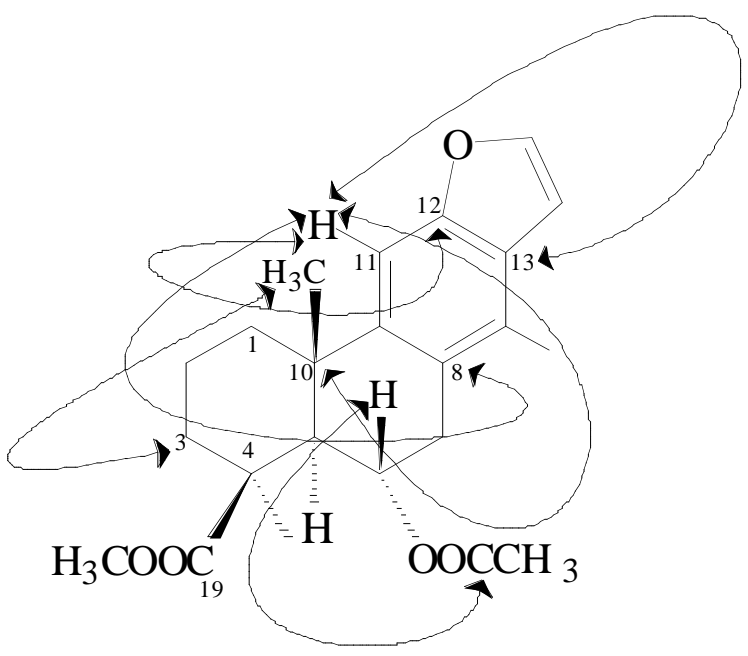

Figure 2: $\mathrm{HMBC}$ correlations for benthaminin 3.

\subsubsection{Antibacterial Effects of Benthaminin 3}

Bioactivity studies of the compound revealed that it possessed antibacterial activity against ten Gram positive and Gram negative bacteria, including three resistant strains namely, methicillin-resistant $S$. aureus, tetracyline-resistant $S$. aureus and erythromycinresistant $S$. aureus. In all cases with MIC values were greater than $1000 \mu \mathrm{g} / \mathrm{mL}$ (Table 2). Benthaminin 3 was however more active against Staphylococcus aureus and Bacillus subtilis with an MIC of $63 \mu \mathrm{g} / \mathrm{mL}$ (Table 2). Microbial infections and the presence of oxygen free radicals are known impediments to wound healing [12]. Notable among the micro-organisms delaying or inhibiting wound healing are Staphylococcus, Streptococcus and Pseudomonas species [13]. On the basis of the antibacterial properties demonstrated by benthaminin 3 as well as other antibacterial and antioxidant cassane-type diterpenoid compounds isolated from the petroleum spirit extract of the root bark of this plant [8], the traditional use of the lipophilic preparations of these species is supported.

\section{CONCLUSION}

In the current study, a novel compound designated benthaminin 3 , a cassane-type furanoditerpenoid compound possessing antibacterial activity has been isolated from the chloroformic extract of $C$. benthamiana. To the best of our knowledge, this represents the first report of the isolation of this compound from $C$. benthamiana.

\section{EXPERIMENTAL}

\subsection{Materials, Methods and Techniques}

\subsubsection{Plant Materials}

The root bark of $C$. benthamiana (Baill.) Herend. and Zarucchi was collected from Akwapim Mampong in

Table 2: Minimum Inhibitory Concentration (MIC) $(\mu \mathrm{g} / \mathrm{mL})$ of Compound on Test Organisms

\begin{tabular}{|c|c|c|}
\hline Test Organism & Strain & Benthaminin 3 \\
\hline \hline Methicillin-resistant S. aureus & SA 1199B (NorA) & $>1000$ \\
\hline Tetracycline-resistant S. aureus & XU 212 (Tek) & $>1000$ \\
\hline Erythromycin-resistant S. aureus & RN 4220 (MsrA) & 63 \\
\hline S. aureus (Wild) & NCTC 4263 & 63 \\
\hline B. subtilis & NCTC 10073 & 250 \\
\hline S. faecalis & NCTC 775 & 125 \\
\hline P. aeruginosa & NCIMB 1042 & 250 \\
\hline M. flavus & NCTC 7743 & $>1000$ \\
\hline M. fortuitum & ATCC 6841 & 25 \\
\hline M. smegmatis & ATCC 14468 & \\
\hline
\end{tabular}

All experiments were carried out in triplicate. $200 \mu \mathrm{g} / \mathrm{mL}$ of tetracycline and rifampicin served as positive controls $(\mathrm{MIC}=20 \mu \mathrm{g} / \mathrm{mL}) ; \mathrm{n}=3$. 
the Eastern region of Ghana in August, 2003. It was authenticated by Mr H R Blagogee, a taxonomist at the Centre for Scientific Research into Plant Medicine (CSRPM), Mampong Akwapim, Ghana, where voucher specimen \#RADMB11 is deposited.

\subsubsection{Extraction and Isolation}

$2.7 \mathrm{Kg}$ (dry weight) of coarsely powdered root bark of $C$. benthamiana were extracted with chloroform (5L) using a Soxhlet apparatus for $24 \mathrm{~h}$. The extract was concentrated under reduced pressure at $40^{\circ} \mathrm{C}$ to dryness to obtain a dark-brown syrup [20.9g (1.1\%) yield]. This extract was subjected to VLC on silica gel $1000 \mathrm{~g}$, eluted initially with chloroform (2L) and subsequently with chloroform: $\mathrm{MeOH}$ 95:5 (6L), 9:1 (9L), 4:1 (4L), 3:2 (5L), 2:3 (2L) and finally with $\mathrm{MeOH}$ (3L). The TLC profiles [Give TLC system used] of aliquots collected enabled six major fractions to be bulked to give MCF1 (1.3g), MCF2 (2.8g), MCF3 $(9.70 \mathrm{~g})$, MCF4 $(0.97 \mathrm{~g})$, MCF5 (2.63g) and MCF6 $(2.05 \mathrm{~g})$. These were tested for antibacterial activity (The lowest MIC values for the various fraction were as follows: MCF1 $(>1000 \mu \mathrm{g} / \mathrm{mL}$ against all test organisms), MCF2 (125 $\mu \mathrm{g} / \mathrm{mL}$ against $S$. aureus), MCF3 (>1000 $\mu \mathrm{g} / \mathrm{mL}$ against all test organisms), MCF4 (250 $\mu \mathrm{g} / \mathrm{mL}$ against $B$. subtilis and $M$. flavus), MCF5 $(>1000 \mu \mathrm{g} / \mathrm{mL}$ against all test organisms) and MCF6 $(250 \mu \mathrm{g} / \mathrm{mL}$ against $B$. subtilis, $M$. flavus and $S$. faecalis) ) and the most active fraction MCF2 was further fractionated using CC SG/ chloroform:MeOH gradient to yield fractions MCF21-MCF25. MCF25 was subjected to column chromatography on silica gel $(400 \mathrm{~g})$, eluting with chloroform and gradually increasing the polarity of the solvent by $1 \%$ increments of $\mathrm{MeOH}$. Aliquots were monitored on TLC and fractions showing a single zone were combined and pure compound $\mathrm{RC} 4$, later designated benthaminin $\mathbf{3}$, isolated after a final preparative TLC separation.

\subsubsection{Chromatographic Investigation}

\subsubsection{Analytical TLC}

Precoated aluminium-backed silica Gel $F_{254}$ TLC plates $(0.25 \mathrm{~mm}$ thickness), product code, OB481662, were purchased from Merck KGaA, Germany. Suitable solvent systems were used and acidic anisaldehyde used as detecting reagent.

\subsubsection{Preparative TLC (PrepTLC)}

The plates were prepared by mixing $90 \mathrm{~g}$ of silica gel $60 \mathrm{PF}_{254}$, product code, TA 1349747 525, for preparative thin-layer chromatography (Merck KGaA, Germany), with $260 \mathrm{ml}$ of distilled water. The slurry was spread onto 5 glass plates with the aid of a spreader to give $1.0 \mathrm{~mm}$ thickness. After drying at room temperature, the plates were activated at $105^{\circ} \mathrm{C}$ for 2 hours. Zones were detected under UV $254 \mathrm{~nm}$ as quenching zones, or showed florescence at $366 \mathrm{~nm}$, scraped off, eluted with the solvent system: chloroform: petroleum spirit (7:3), filtered and the resulting solutions were evaporated to dryness under reduced pressure. Analytical TLC was then used to check the level of purity of the compounds. Impure samples were subjected to further preparative TLC analysis until a pure sample was obtained.

\subsubsection{Vacuum Liquid Chromatography (VLC)}

The dry method of packing was used in this instance. The column was packed by putting silica gel 60 (0.040-063mm), product code TA 1280185 438, for column chromatography (Merck KGaA, Germany) in a Buchner funnel attached to a $1000 \mathrm{~L}$ conical flask with a side arm connected to a vacuum supply. The sample was triturated with an equal weight of silica gel $60(4.2 \mathrm{~g}$ and $40.9 \mathrm{~g}$ for preliminary and bulk experiments respectively), and applied to the top of the column. A thin film of non absorbent cotton wool was spread evenly on top of the column to prevent disturbance to the surface of the column when the eluant was being added. The eluant was added at a fixed volume of $50 \mathrm{~mL}$ and $200 \mathrm{~mL}$ respectively for both preliminary and bulk experiments, gradually and carefully. Vacuum was then applied and the eluant was sucked through the column by reduced pressure into the collecting conical flask. Fractions (between $100-200 \mathrm{~mL}$ ) were collected as aliquots of eluants of one polarity. Low polarity eluants were used initially and the polarity gradually increased. At any given polarity, the eluant was continually added and aliquots collected until no compounds were being desorbed from the column into the collectors, this being detected by TLC monitoring. After elution of compounds ceased, the polarity of the eluant was increased and the entire process repeated until finally $100 \%$ of the most polar solvent was used to wash the column. For the preliminary VLC analysis, a smaller Buchner funnel $(10 \mathrm{~cm} \times 8 \mathrm{~cm})$, was employed using $400 \mathrm{~g}$ of silica gel. The bulk of the VLC was done using $1500 \mathrm{~g}$ of silica gel in a bigger Buchner funnel $(25 \mathrm{~cm} \times 20 \mathrm{~cm})$. In all cases, based on the TLC profiles of aliquots collected, similar fractions were bulked and the solvents recovered using a rotary evaporator under reduced pressure. Bioactivity studies were carried out on all fractions to determine which fractions possessed activity. All active fractions were further subjected to column chromatography (CC) separations, and other techniques, to isolate the active compounds 
responsible for any observed activity. Since VLC is a quick and crude way of dividing the crude extracts into relatively smaller units, larger aliquot volumes are collected, unlike in $\mathrm{CC}$, where aliquots collected are in relatively small volumes.

\subsubsection{Column Chromatography}

The wet method of packing was employed here. Column was packed with silica gel 60 (0.040$0.063 \mathrm{~mm}$ ), product code TA1280185438, for Column Chromatography (Merck KGaA, Germany), wetted with the initial eluting solvent of the lowest polarity. The sample was triturated with equal amount of silica gel and applied to the top of the column. After the sample was adsorbed on top of the column, eluants of increasing polarities were added in fixed volumes. Fractions were eluted from the column under gravity and collected in glass specimen tubes. As in VLC, the TLC profiles of aliquots collected were monitored by analytical TLC, by observing under UV at both 254 and $366 \mathrm{~nm}$ and then spraying with acidic anisaldehyde reagent.

\subsubsection{Determination of Minimum Inhibitory Concentration (MIC)}

Stock solutions were prepared by dissolving $4 \mathrm{mg}$ RC4 of the compound in $80 \mu$ l of DMSO. Sterile water was added with slight heating to aid dissolution and the volume made up to $2 \mathrm{ml}$ in a sterile bottle. This was then sonicated to ensure complete dissolution. The stock mixture was passed through a $0.2 \mu \mathrm{m}$ pyrogenic filter to sterilize the solution and serially diluted to arrive at concentrations between $1000 \mu \mathrm{g} / \mathrm{ml}$ and $7.8 \mu \mathrm{g} / \mathrm{ml}$. The inocula of micro-organisms were prepared from broth cultures, and serial dilutions were made to achieve a suspension of approximately $10^{5} \mathrm{CFU} / \mathrm{ml}$. For every experiment, a sterility check ( $2 \%$ DMSO and medium), negative control ( $2 \% \mathrm{DMSO}$, medium and inoculum), and positive control ( $2 \%$ DMSO, medium, inoculum and water-soluble antibiotic) were included. The 96-well plates were prepared by dispensing into each well $100 \mu \mathrm{L}$ each of double strength nutrient broth, $100 \mu \mathrm{L}$ of test solutions and $20 \mu \mathrm{l}$ of the inoculum. Contents of each well were thoroughly mixed with a sterile-tipped multi-channel pipette and the plates incubated at $37^{\circ} \mathrm{C}$ for 24 hours. Any growth of the micro-organisms was determined by adding $20 \mu \mathrm{l}$ of a $5 \%$ solution of tetrazolium salt (MTT) and incubating for a further 30 minutes. Dark coloured wells indicated growth whilst colourless wells indicated inhibition of growth of organisms. $200 \mu \mathrm{g} / \mathrm{ml}$ tetracycline served as a positive control. All experiments were performed in triplicate.

\subsubsection{Micro-Organisms Used}

The following bacterial strains obtained from the UK National Culture Collection and School of Pharmacy, University of London, were used in the bioassay: Micrococcus flavus (NCTC 7743), Bacillus subtilis (NCTC 10073), Staphylococcus aureus (NCTC 4163), Mycobacterium fortuitum (ATCC 6841), Mycobacterium smegmatis (ATCC 14468), multidrug-resistant $S$. aureus SA-1199B, tetracycline-resistant $S$. aureus XU 212, erythromycin-resistant $S$. aureus RN 4220, Streptococcus faecalis (NCTC 775) and Pseudomonas aeruginosa (NCIMB 10421).

\section{AUTHENTIC REFERENCE}

\subsection{Antibiotics}

Tetracycline and rifampicin were obtained from Sigma Aldrich Co Ltd. UK

\subsection{Chemicals}

Unless otherwise specified, chemicals were analytical grade and purchased from Sigma Aldrich Co Ltd. UK. Organic solvents were analytical grade and purchased from BDH Laboratory Supplies, UK.

\subsection{Apparatus}

Low resolution ESI mass spectra were run on a QToF (Waters) instrument. The NMR spectra were obtained on a Bruker 500 spectrometer with chemical shifts reported in $\delta(\mathrm{ppm})$ using TMS as an internal standard.

\section{REFERENCES}

[1] Herendeen PS, and Zarucchi JL. Validation of Caesalpinia subgenus Mezoneuron (Desf.) vidal and new combinations in Caesalpinia for two species from Africa. Ann Missouri Botanical Garden 1990; 77: 854-55.

http://dx.doi.org/10.2307/2399679

[2] Irvine FR. Woody Plants of Ghana. Oxford University Press, London 1961; pp. 312-313.

[3] Abbiw D. Useful Plants of Ghana. Intermediate Technical Publications and Royal Botanic Gardens, Kew, 1990; 199200.

[4] Attah Y. Personal communication 2003.

[5] Banskota F, Attamimi TZ, Usia YT, Linn SK, Kadota S. Novel norcassane-type diterpene from the seed kernels of Caesalpinia crista. Tetrahedron Lett 2003; 6879-82. http://dx.doi.org/10.1016/S0040-4039(03)01722-2

[6] Jiang RW, Paul PHB, Shuang-Cheng MA, Thomas CWM. New antiviral cassane furanoditerpenes from Caesalpinia minax. J Nat Prod 2001; 1266-1272.

http://dx.doi.org/10.1021/np010174+

[7] Mbagwu HOC, Anene RA, Adeyemi OO. Anti-diarrhoeal activity of the aqueous extract of Mezoneuron benthamianum Baill (Caesalpiniaceae). J Ethnopharmacol 2008; 16-20. http://dx.doi.org/10.1016/j.jep.2007.10.037 
[8] Dickson RA, Houghton PJ, Hylands, PJ. Antibacterial and antioxidant cassane diterpenoids from Caesalpinia benthamiana. Phytochemistry 2007; 1436-41. http://dx.doi.org/10.1016/j.phytochem.2007.03.008

[9] Kitagawa I, Simanjuntak P, Mahmud T, et al. Indonesian medicinal plants.XIII: chemical structures of caesaldekarins $\mathrm{c}, \mathrm{d}$, and e, three additional cassane-type furanoditerpenes from the roots of Caesalpinia major (Fabaceae). Several interesting reaction products of caesaldekarin a provided by $\mathrm{N}$-bromosuccinimide treatment. Chem Pharmaceut Bull 1996; 1157-61.

http://dx.doi.org/10.1248/cpb.44.1157

[10] King FE, Godson DH, King TJ. The chemistry of extractives from heartwoods. J Chem Soc 1955; 1117-19.
[11] Peter S, Tinto WF, McLean S, Reynolds WF, Yu M. Cassane diterpenes from Caesalpinia bonducella. Phytochemistry 1998; 1153-55.

[12] Houghton PJ, Hylands PJ, Mensah AY, Hensel A, Deters AM. In- vitro tests and ethnopharmacological investigations: Wound healing as an example. J Ethnopharmacol 2005; 100: 100-107.

http://dx.doi.org/10.1016/i.jep.2005.07.001

[13] Thomas S. Wound Management and Dressings. The Pharmaceutical Press London 1990; 6-7. 